\title{
Association of Psychological Health and Perceived Oral Health in Elderly Individuals: Focusing on Depression, Stress, and Suicidal Ideation
}

\author{
Hyun-Ok Lee ${ }^{\dagger}$, Sun-Mi Kim, and Ji-Young Park \\ Department of Dental Hygiene, Wonkwang Health Science University, Iksan 54538, Korea
}

The present study aimed to examine the influence of mental health factors on oral health factors using data from the Korean National Health and Nutrition Examination Survey (KNHANES). A total of 1,548 elderly individuals aged over 65 years who completed the screening and health questionnaire were selected as the final study participants. The psychological health factors related to perceived oral health status were depression and stress when the gender was controlled, and in terms of gender, both elderly male and female individuals were stressed and depressed. These results suggested that the psychological health of elderly individuals negatively affects not only perceived oral health but also the quality of life. In addition, the psychological health of the elderly individuals should be systematically managed along with oral health care and overall health care and lifestyle. If a comprehensive health management system is available for low-income groups or an elderly person living alone, it should positively affect and improve the quality of life of elderly individuals.

Key Words: Aged, Mental health, Oral health status

\section{Introduction}

The aging of population is occurring rapidly given the population distribution characteristics of Korea. Thus, with the rapid growth of the elderly population, elderly individuals will account for $40.1 \%$ of the entire population by $2060^{1)}$. As such, successful aging and policies on the health management of elderly individuals have been a topic of interest, which help lengthen the lifespan of the rapidly growing elderly population. The period of old age is marked by several important health concerns, such as weakening of physical functions, disorders related to old age, and changes in social and psychological disposition and mental adaptation, and these problems commonly cause depression $^{2,3)}$, which is a common psychological response to negative emotions and losses, such as death of a spouse or other loved one, economic instability, and psychological changes after decreased motor functions ${ }^{3)}$
The inability to perceive depression as a problem may lead to health issues, such as physical, cognitive, and social disorders, which decrease the quality of life and increase the risk of suicide ${ }^{2)}$ and sleeping disorders ${ }^{3)}$.

Despite such rapid social changes, the mortality rate of suicide in Korea remains the highest among the member countries of the Organisation for Economic Co-operation and Development. The rates of successful suicide are $8.0 \%$ for individuals below 65 years and $31.8 \%$ for elderly individuals over 65 years. The average number of suicides per 100,000 population were 42.4 for individuals aged 60 years, 73.1 for individuals aged 70 years, and 104.5 for individuals aged 80 years ${ }^{4}$. In other words, the success rates of suicides in elderly individuals was high. In addition, the possibility of suicide attempts increases with age. Thus, the identification of prevention methods by first recognizing suicide-related factors as early as possible is important ${ }^{5}$. Moreover, the psychological health of elderly 
individuals is influenced by demographic, economic, physical, and psychological factors, such as stress and sleep duration. In addition, it significantly affects behavior or health status ${ }^{5)}$. However, numerous studies consider elderly individuals as a homogeneous group and tend to overlook the diversity within the group, indicating the need for a specific and differentiating study that specifies demographic or socioeconomic factors ${ }^{2,3}$.

With the perception that quality of life affects oral health, the perception of perceived oral health and its objectives should be improved $^{6)}$. Perceived oral health is easier to use in projects related to oral health compared to the traditionally used objective and can be an accurate predictor of oral health since it measures the perceived level of oral health when an individual predicts his/her own oral health. Moreover, it can be used as evaluation criteria for the burden of oral diseases and a useful summary indicator ${ }^{7)}$. Finlayson et al. ${ }^{7)}$ reported that higher levels of stress lead to higher levels of perceived oral health. Ma and $\mathrm{Cho}^{8)}$ identified that stress and depression influence the oral health of adolescents. Moreover, Won and $\mathrm{Kim}^{9)}$ studied the perceived oral health of adults, which is associated with mental counselling, higher levels of stress, depression, and presence of suicidal ideation. These studies have generally focused on adults and adolescents, and studies on elderly individuals are limited.

Thus, the present study used the raw data from Korean National Health and Nutrition Examination Survey (KNHANES) to identify the association between the perceived oral health of elderly individuals and various factors that affect psychological health, such as stress, depression, and suicidal ideation in terms of gender. In addition, the results were presented as basic data that improve oral and psychological health.

\section{Materials and Methods}

\section{Subjects}

This study has utilized the raw data of the 6th KNHANES conducted in 2015, which are used for goal setting to improve the health of citizens and develop and evaluate health projects through the statistics of the general health levels, health-related behaviors, and food and nutrition intake of the population ${ }^{10)}$. These data are obtained from the health, check-up, and nutrition survey $^{10)}$. A total of 1,548 elderly individuals aged over 65 years who have completed the health survey were included in the study. Based on a study by Won and $\mathrm{Kim}^{9}$, the independent variables included gender (male and female), age $(65 \sim 69,70 \sim 74,75 \sim 79$, and over 80 years old) and the presence of a spouse (yes or no). The socioeconomic characteristics included the level of education (no education, elementary school, middle school, or over), economic activity (yes or no), and household income (high, medium high, medium low, and low). Factors that affect mental health included symptoms of depression (depression for more than 2 weeks or none), stress perception (low and high), and experience of suicidal ideation (yes or no). The dependent variable of oral health was measured through face-to-face interviews on perceived oral health and reclassified as very good, good, average as "good" and bad, and very bad as "bad."

\section{Methods}

KNHANES has a complex sample design where sample extraction methods determine representation, and data were analyzed using yearly weights. The domain variables were analyzed using complex sample analysis methods, and the stated values (n) were actual observed values and percentages that are reflected as sample weights. For the general characteristics, descriptive statistics and frequency analysis were used. The relationship between demographic statistics and psychological health was analyzed using chi-squared tests. The influence of psychological health on perceived oral health that depend on the participant's psychological health, perceived oral health, and gender was analyzed through logistic regression analysis. OR and 95\% CI were calculated, and a p-value of 0.05 was considered statistically significant. STATA 11.0 SE (Stata Co., College Station, TX, USA) was used in the analysis. This study was passed at Wonkwang University with IRB exemption (WKIRB-201705-SB-023). 


\section{Results}

\section{Demographic characteristics}

The participants were aged between 65 and 69 years. The majority of male participants were married, and the majority of female participants were unmarried. In terms of education level, most of the participants in the group answered "none." In terms of income, low-level income was significant irrespective of gender, and with regard to economic activity, most participants did not participate in economic activities. In terms of smoking, the male participants indicated that they were former smokers. As to alcohol consumption, most of the participants in the group indicated they drank more than twice a month. Most of female participants are

Table 1. Distribution and Rate of Study Subjects by Demographic Characteristics $(n=1,548)$

\begin{tabular}{lcc}
\hline \multicolumn{1}{c}{ Characteristic } & Male $(\mathrm{n}=673)$ & Female $(\mathrm{n}=875)$ \\
\hline Age $(\mathrm{y})$ & & \\
$65 \sim 69$ & $246(36.6)$ & $263(29.1)$ \\
$70 \sim 74$ & $180(27.3)$ & $254(29.7)$ \\
$75 \sim 79$ & $151(22.2)$ & $205(23.8)$ \\
$\geq 80$ & $96(14.3)$ & $153(17.2)$ \\
Living with spouse & & \\
Yes & $580(86.1)$ & $401(45.8)$ \\
No & $93(13.9)$ & $473(54.2)$ \\
Education & & \\
Illiteracy & $239(42.8)$ & $565(76.4)$ \\
Elementary school & $98(16.0)$ & $76(9.6)$ \\
$\geq$ Middle school & $239(41.2)$ & $98(13.7)$ \\
Household income & & \\
Low & $261(38.5)$ & $432(49.5)$ \\
Middle-low & $212(31.8)$ & $223(25.6)$ \\
Middle-high & $119(18.7)$ & $138(16.9)$ \\
High & $74(11.0)$ & $72(7.8)$ \\
Employment & & \\
Employed & $251(43.5)$ & $185(25.0)$ \\
Unemployed (housewife, & $326(56.5)$ & $559(74.9)$ \\
$\quad$ other) & & \\
Smoking status & $194(31.7)$ & $489(63.2)$ \\
Never & $56(9.1)$ & $146(18.8)$ \\
Former & $362(59.2)$ & $142(18.0)$ \\
Current & & \\
Number of alcoholic drinks in past year & & \\
None & & \\
$\leq 1$ per month & & \\
$\geq 2$ per month & & \\
\hline
\end{tabular}

Values are presented as $\mathrm{n}(\%)$. Percentages (\%) reflect sample weights. non-smokers and non-drinkers (Table 1).

\section{Relationship between demographic characteristics and psychological health}

In terms of stress, statistically significant differences were observed in male participants who had spouses and income and in female participants who had income ( $\mathrm{p}$ $<0.05$; Table 2). In terms of depression symptoms, no significant differences were observed in male participants who had spouses $(p<0.001)$ and income and those who participated in economic activities $(p<0.01)$. In the case of female participants, statistically significant differences were observed in terms of income $(\mathrm{p}<0.01)$. With regard to suicidal ideation, significant differences were noted in male participants in terms of age, marital status, income, and economic activity $(\mathrm{p}<0.001)$. Statistical differences were also observed in female participants in terms of age $(\mathrm{p}<0.01)$ and economic activity $(\mathrm{p}<0.05)$.

\section{Influence of the psychological health of patients on perceived oral health}

To observe the influence of psychological health on perceived oral health, a logistic regression analysis was conducted after adjusting for gender, age, level of education, presence of spouse, income, and economic activity (Table 3 ). The results indicated that depression and stress influenced the perceived oral health of patients. The perceived oral health was 1.60 times higher in participants who continuously experienced depression for more than 2 weeks versus those who did not and 1.70 times higher in participants who were stressed versus those who were not $(p<0.05)$.

\section{Influence of the psychological health of patients on perceived oral health in terms of gender}

To observe the influence of psychological health on perceived oral health in terms of gender, a logistic regression analysis was conducted after adjusting for age, level of education, presence of spouse, income, and economic activity (Table 4). Depression affected the perceived oral health of male participants, and depression and stress influenced the perceived oral health of female participants. The perceived oral health was 1.60 times 


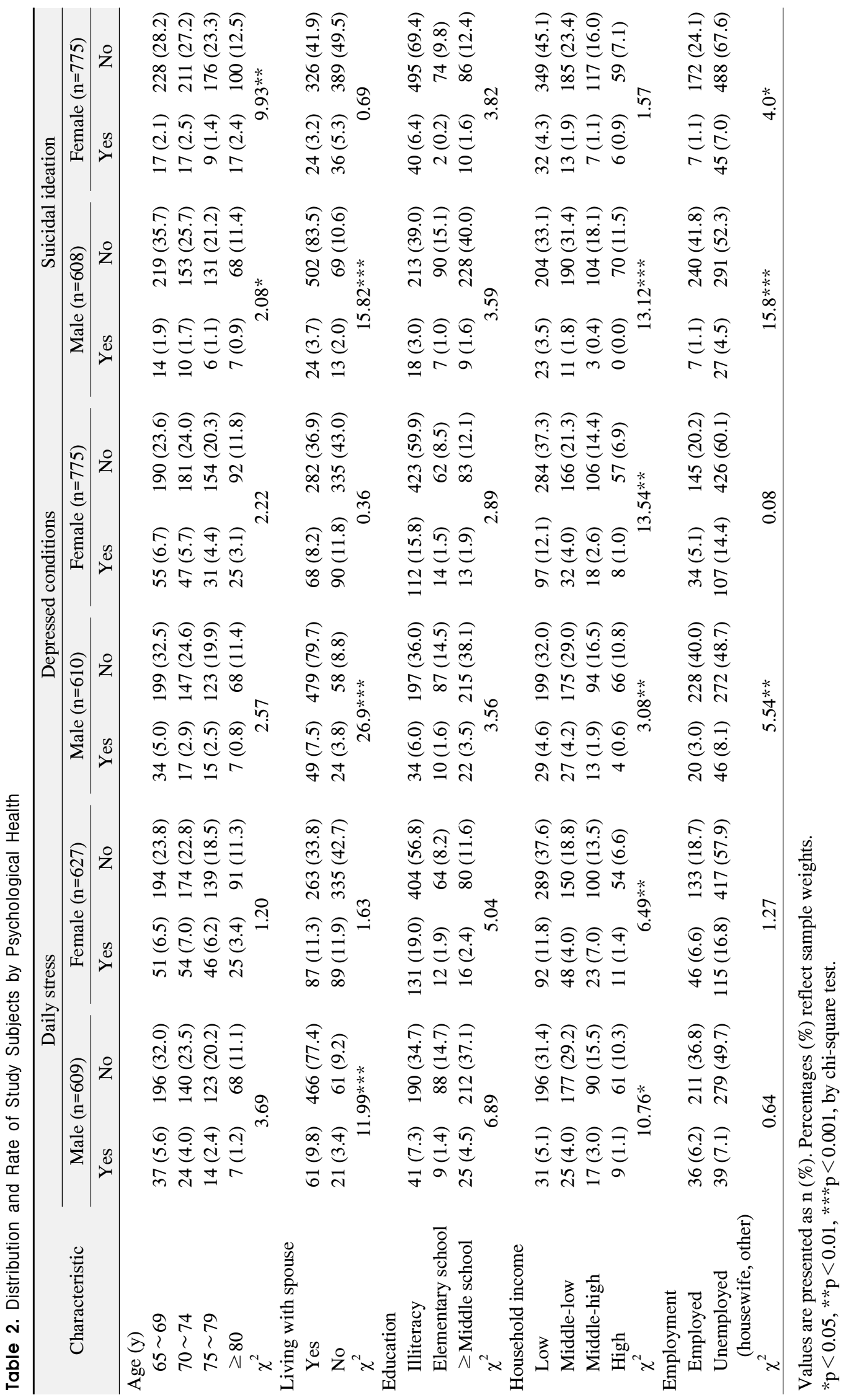


higher in male participants who reported depression versus those who did not, and perceived oral health was 2.46 times higher in female participants who reported depression versus those who did not $(\mathrm{p}<0.05)$. Moreover, the OR of female participants who were stressed, causing a low perception of oral health, was 1.39 times higher than those who were not $(\mathrm{p}<0.05)$.

\section{Discussion}

Despite the fact that the importance and perception of

Table 3. Association between Psychological Health and Perceived Oral Health Status

\begin{tabular}{lccccc}
\hline & \multicolumn{5}{c}{ Perceived oral health status } \\
\cline { 2 - 6 } Variable $^{\mathrm{a}}$ & $\begin{array}{c}\text { Good } \\
(\mathrm{n}=586)\end{array}$ & $\begin{array}{c}\text { Bad } \\
(\mathrm{n}=681)\end{array}$ & OR & $95 \% \mathrm{CI}$ & $\mathrm{p}>\mathrm{t}^{\mathrm{b}}$ \\
\hline \multicolumn{7}{c}{ Depressed conditions } \\
$\quad$ No & $509(40.7)$ & $552(43.3)$ & Ref. & & \\
Yes & $77(5.8)$ & $129(10.0)$ & 1.60 & $1.06 \sim 2.40$ & 0.020 \\
Daily stress & & & & & \\
No & $497(41.5)$ & $535(39.4)$ & Ref. & & \\
Yes & $88(7.1)$ & $146(11.9)$ & 1.70 & $0.49 \sim 1.00$ & 0.044 \\
Suicidal ideation & & & & \\
$\quad$ No & $550(43.6)$ & $633(49.3)$ & Ref. & & \\
$\quad$ Yes & $35(2.8)$ & $48(4.0)$ & 1.29 & $0.75 \sim 2.22$ & 0.352 \\
\hline
\end{tabular}

Values are presented as $\mathrm{n}(\%)$. Percentages (\%) reflect sample weights.

OR: odds ratio, $95 \% \mathrm{CI}$ : $95 \%$ confidence interval, ref.: reference. ${ }^{a}$ Adjusted for sex, age, education, living with spouse, household income and employment. ${ }^{\mathrm{b}} \mathrm{By}$ logistic regression. psychological health of elderly individuals have been a topic of interest worldwide, Korea has generally experienced a low level of perception, and policies on the psychological health of elderly individuals are limited ${ }^{11)}$. In a rapidly aging population, the use of effective methods that will improve the psychological health of elderly individuals is important. Thus, this study has used data obtained from KNHANES to observe elderly individuals aged over 65 years and identify the relationship between perceived oral health and psychological health according to gender. In addition, this study aimed to present methods that improve the health of elderly individuals and data that can be used for projects for the elderly individuals.

In terms of the association between the general characteristics of the elderly individuals and their psychological health, the occurrence of stress, depression, and suicidal ideation was high in male participants who had low income. Female participants with low income had high levels of stress and depression. These results were in accordance with the study of Ross and $\mathrm{Wu}^{12)}$, which indicated that elderly individuals with higher socioeconomic status had short durations of chronic diseases and did not usually feel depressed. Moreover, the results of this study with regards to income are in accordance with the study conducted by Cho and Choi ${ }^{13)}$, which shows that low-income classes had higher levels of depression and stress along with groups with low income and lack of retirement plans ${ }^{14)}$. Therefore, a national level policy on

Table 4. Association between Psychological Health and Perceived Oral Health by Gender

\begin{tabular}{|c|c|c|c|c|c|c|c|c|c|c|}
\hline \multirow{3}{*}{ Variable $^{\mathrm{a}}$} & \multicolumn{10}{|c|}{ Perceived oral health status } \\
\hline & \multicolumn{5}{|c|}{ Male } & \multicolumn{5}{|c|}{ Female } \\
\hline & Good & $\mathrm{Bad}$ & OR & $95 \% \mathrm{CI}$ & $\mathrm{p}>\mathrm{t}^{\mathrm{b}}$ & Good & $\mathrm{Bad}$ & OR & $95 \% \mathrm{CI}$ & $\mathrm{p}>\mathrm{t}^{\mathrm{b}}$ \\
\hline \multicolumn{11}{|c|}{ Depressed conditions } \\
\hline No & $235(42.6)$ & $261(45.6)$ & Ref. & & & $274(39.3)$ & $291(41.5)$ & Ref. & & \\
\hline Yes & $21(3.3)$ & $47(8.5)$ & 1.60 & $1.06 \sim 2.40$ & 0.021 & $56(7.9)$ & $82(11.3)$ & 2.46 & $0.37 \sim 4.41$ & 0.003 \\
\hline \multicolumn{11}{|l|}{ Daily stress } \\
\hline No & $227(40.8)$ & $260(45.5)$ & Ref. & & & $270(38.4)$ & $275(38.4)$ & Ref. & & \\
\hline Yes & $28(5.0)$ & $48(8.7)$ & 1.05 & $0.90 \sim 1.57$ & 0.232 & $60(8.8)$ & $98(14.4)$ & 1.39 & $1.07 \sim 1.80$ & 0.046 \\
\hline \multicolumn{11}{|l|}{ Suicidal ideation } \\
\hline No & $242(43.7)$ & $287(50.5)$ & Ref. & & & $308(43.6)$ & $346(48.5)$ & Ref. & & \\
\hline Yes & $13(2.1)$ & $21(3.7)$ & 0.85 & $0.49 \sim 1.48$ & 0.565 & $22(3.6)$ & $27(4.3)$ & 1.41 & $0.70 \sim 2.85$ & 0.335 \\
\hline
\end{tabular}

Values are presented as $\mathrm{n}(\%)$. Percentages $(\%)$ reflect sample weights.

OR: odds ratio, $95 \%$ CI: $95 \%$ confidence interval, ref.: reference.

${ }^{\mathrm{a}}$ Adjusted for age, education, living with spouse, household income and employment. ${ }^{\mathrm{b}}$ By logistic regression. 
job creation and medical cost burdens would reduce economic problems and help improve the quality of life, resulting to positive influences on psychological health.

Male participants had higher levels of depression, stress, and suicidal ideation in the absence of their spouses, which was in accordance with the study of Hur and $\mathrm{Yoo}^{15)}$ showing that the presence or absence of a spouse influence the psychological health of male elderly individuals. The presence of a spouse is important in the psychological health of male elderly individuals, and participants without a spouse may experience higher levels of solitude, separation, and financial hardship compared to those with spouses, which influenced their psychological health. Thus, the use of methods that improve psychological health and prevent elderly individuals to feel isolated or withdrawn from society is important.

Suicide incidence among elderly individuals are thought and planned over long periods of time and associated with fatal methods. Thus, it is associated with death. However, despite the fact that elderly individuals comprise a group with a high risk for suicide, suicide incidence among elderly individuals is often ignored ${ }^{16)}$. The causes include the dishonoring nature of suicide, and the discussion of suicide itself among elderly individuals is not part of the traditional social value of upholding elders ${ }^{16)}$. Moreover, depression as a factor of suicide among elderly individuals is often discussed and directly and indirectly influences suicidal ideation. Therefore, a comprehensive management by the regional mental health centers is extremely important, the incidence and graveness of depression should not be overlooked. This study indicated that age of both male and female participants was associated with suicidal ideation. Comparatively, younger-old elderly individuals are more likely to have resident family members compared to older-old elderly individuals. The presence of resident family members may lead to restrictions in movement. However, the presence and support of these family members would likely motivate elderly individuals, thus preventing suicidal ideation. Moreover, restrictions in activity, pain, and discomfort with increasing age may possibly cause suicidal ideation $^{17)}$. Thus, future studies should include chronic diseases and social support.

The results of this study indicated that stress and depression as the sub-factors of psychological health were found to affect perceived oral health. These results are in accordance with the research of Chun and Jung ${ }^{18)}$. stress and depression leads to decreased chewing ability or dry mouth because of aging along with poor teeth management that might continue for a long period of time and eventually will lead to teeth loss and oral diseases. Thus, the development of oral health programs for elderly individuals along with psychological health is important.

Stress and depression influenced perceived oral health in terms of gender. Specifically, stress and depression were confirmed to influence perceived oral health of the female participants. Elderly women are more stressed due to solitude, depression, and isolation compared to elderly men. Less social participation causes these women to be at risk socioeconomically compared to men, and the cost associated with general healthcare and oral health management is the cause. Thus, education should be provided to these women so that they will have a positive attitude towards accepting teeth loss as part of aging, and they should be taught that the absence of treatment will worsen oral health. Moreover, elderly women are not careful with their health. Since overall health is also related to oral health, health education should consistently be provided, which includes the importance of regular oral check-ups.

Depression affects the perceived oral health of both elderly men and women. These results are in accordance with the study by Chun and Jung ${ }^{18)}$. Therefore, depression should be considered in reviewing policies on the oral health of elderly individuals. Future studies should include comprehensive factors that affect demographic, physical, psychological, social, and economic factors, and a multi-dimensional study on depression in terms of gender should be considered. Moreover, comprehensive counselling programs that include psychological health factors should be provided in addition to regular check-ups and appropriate education in oral health improvement programs, thus changing the perception and attitude towards oral and psychological health.

This study is significant because elderly individuals 
were classified based on gender to confirm the relationship between psychological health and perceived oral health while considering confounding factors. On the other hand, the limitations of this study include the data obtained from KNHANES. Though the information has secured representability and reliability, still the causal relationships were not explained since the study was cross sectional. Future studies should investigate various factors, such as social support, chronic diseases, and health activities that are relevant to the psychological health of elderly individuals. Moreover, prior to the development of oral health programs for the elderly individuals, personalized health education should include oral health management for low-income elderly individuals and programs for the management of psychological health.

\section{Acknowledgements}

This paper was supported by a 2017 research fund support from a Wonkwang Health Science University.

\section{References}

1. Statistics Korea: Population projections 2010-2060. Statistics Korea, Daejeon, 2010.

2. Yang SA: Factors influencing depression of elderly women in a metropolitan city. J Korean Pubilc Health Nurs 26: 158-173, 2012.

3. Park SY: A study on depression, ADL, IADL, and QOL among community-dwelling, low income elderly. J Korean Public Health Nurs 3: 78-90, 2009.

4. Statistics Korea: Cause of death statistics. Retreived August 2 2017, from http://kostat.go.kr/portal/korea/kor_nw/2/6/2/index. board bmode $=$ read $\&$ aSeq $=308559 \&$ pageNo $=1 \&$ row $N u m=1$ $0 \&$ navCount $=10 \&$ currPg $=\&$ sTarget $=$ title $\&$ s Txt $=(2014$, June $10)$.

5. Kim EK: The risk factors of suicidal ideation by age groups among the elderly in Korea. Korean J Health Promot 15: 54-62, 2015.

6. Boffano P, Roccia F, Pittoni D, Di Dio D, Forni P, Gallesio
C: Management of 112 hospitalized patients with spreading odontogenic infections: correlation with DMFT and oral health impact profile 14 indexes. Oral Surg Oral Med Oral Pathol Oral Radiol 113: 207-213, 2012.

7. Finlayson TL, Williams DR, Siefert K, Jackson JS, Nowjack-Raymer R: Oral health disparities and psychosocial correlates of self-rated oral health in the National Survey of American Life. Am J Public Health 100 Suppl 1: S246-S255, 2010.

8. Ma JK, Cho MJ: Association of sleep time, stress, and depression with the oral health status of Korean adolescents. J Korean Acad Oral Health 40: 178-182, 2016.

9. Won YS, Kim JH: The relationship between psychological health and self-rated oral health on convergence study. J Digit Converg 13: 239-248, 2015.

10. Willits FK, Crider DM: Health rating and life satisfaction in the later middle years. J Gerontol 43: S172-S176, 1988.

11. Lee HK, Sohn MS, Choi M: Factors affecting the mental health of the aged in Korea. J Korean Cont Assoc 12: 672-682, 2012.

12. Ross CE, Wu C: The links between education and health. Am Sociol Rev 60: 719-745, 1995.

13. Cho HA, Choi ES: Association between depression and poor oral health in Korean elderly: the six Korean National Health and Nutrition Examination Survey (KNHANES VI-2). J Korean Soc Dent Hyg 16: 931-941, 2016.

14. Park HM: Effects of oral health behavior and status of elderly people in Korea on mental health and quality of life. Korean J Health Serv Manag 8: 175-185, 2014.

15. Hur JS, Yoo SH: Determinants of depression among elderly person. Ment Health Soc Work 13: 7-22, 2002.

16. Kim HS: The elderly and deviant behavior (suicide). J Welf Aged 10: 25-45, 2000.

17. Szanto K, Gildengers A, Mulsant BH, Brown G, Alexopoulos GS, Reynolds CF 3rd. Identification of suicidal ideation and prevention of suicidal behaviour in the elderly. Drugs Aging 19: 11-24, 2002.

18. Chun JY, Jung ES: Relationship between health factor, oral health factor and prevalence of depression in Korean elderly. J Korean Soc Dent Hyg 15: 963-971, 2015. 